\title{
Management of Breast Hypertrophy: Our Experience
}

\author{
${ }^{1}$ Prema Dhanraj, ${ }^{2} \mathrm{MS}$ Mahesh, ${ }^{3} \mathrm{~N}$ Naveen, ${ }^{4} \mathrm{~K}$ Ramesh Babu, ${ }^{5}$ Rajashekar Jade
}

\begin{abstract}
Breast hypertrophy and sagging of the breast are two different benign disorders of breast that can occur during various stages of women's life from adolescence to menopause. A woman can seek a plastic surgeon for breast reduction for reasons both physical and psychological. Breast reduction surgery or reduction mammoplasty is a plastic surgical procedure by which the sizes of large breasts are reduced. During this procedure, excess skin, fat and breast tissue are removed. The procedure recreates a breast with the desired appearance, contour and volume. Breast lift operation/mastopexy is a procedure where only skin is removed with repositioning of the nipple higher on the chest wall and is the procedure of choice in small but sagging breast.

We present a series of five cases, two presenting with virginal hypertrophy, one with gestational hypertrophy and other two with postmenopausal sagging and hypertrophy. Four patients underwent reduction mammoplasty and one patient underwent mastopexy. None of our patients complained of any problems and were satisfied with the results.
\end{abstract}

Keywords: Breast hypertrophy, Reduction mammoplasty, Mastopexy.

How to cite this article: Dhanraj P, Mahesh MS, Naveen N, Babu KR, Jade R. Management of Breast Hypertrophy: Our Experience. J Med Sci 2015;1(1):21-25.

Source of support: Nil

Conflict of interest: None

\section{INTRODUCTION}

Breast hypertrophy is a benign disorder of the breast in which one or both of the breasts undergo a massive increase in size during adolescence, gestational or postmenopausal age. Sagging of the breast can occur at various stages of women's life like postpartum and lactation, after gaining weight, menopause or at any age. ${ }^{1}$ Many women are genetically predisposed for development

\footnotetext{
${ }^{1}$ Professor and Head, ${ }^{2,3}$ Senior Resident, ${ }^{4}$ Professor

${ }^{5}$ Associate Professor

${ }^{1-4}$ Department of Plastic Surgery, RajaRajeswari Medical College \& Hospital, Bengaluru, Karnataka, India

${ }^{5}$ Department of General Surgery, RajaRajeswari Medical College \& Hospital, Bengaluru, Karnataka, India

Corresponding Author: MS Mahesh, Senior Resident Department of Plastic Surgery, RajaRajeswari Medical College \& Hospital, Bengaluru, Karnataka, India, e-mail: maheshms.6@gmail.com
}

of large breast, whose size increase either by weight gain or pregnancy. Rarely, young women can develop massive and oversized breasts called virginal mammary hypertrophy. ${ }^{2,3}$

\section{INDICATIONS FOR BREAST SURGERY}

A woman can seek a plastic surgeon for breast reduction for reasons both physical and psychological. Breast reduction surgery is done to change the size, weight, firmness and shape of the breasts. A heavy and a pendulous breast themselves may be chronically painful and can cause either neck or back pain or both. The constant pull of heavy breasts may make bra straps leave painful indentations in a woman's shoulders. ${ }^{4}$ Inframammary skin can undergo maceration and its related skin problems. Excessively large breasts can be a cause for troublesome focus of embarrassment for a young girl as well as an elderly woman. Asymmetric unilateral hypertrophy of breast again is a problem from psychological points of view. Heavy breasts reduce the chances of participation in sports or other activities. Some physical activities may be painful or awkward for women who have large breasts. ${ }^{5} \mathrm{It}$ can alter appearance. Large breasts, especially when they are out of proportion to height and weight, can be embarrassing. Teenagers and young women with large breasts may especially feel self-conscious wearing swimsuits and other types of clothing due to unwelcome attention to large breasts. It also may be hard to find clothes that fit well. Thus, the common indications for breast reduction surgery are physical, esthetic and psychological. ${ }^{6,7}$

\section{CONTRAINDICATIONS FOR SURGERY}

Undiagnosed breast lumps or masses, irregular mammography findings and other general medical illnesses.

\section{TREATMENT}

Breast reduction surgery or reduction mammoplasty is a plastic surgical procedure by which the sizes of large breasts are reduced. Considerations are to be paid, in order to retain the function of the breast including the lactational capability and functional sensitivity while carving the breasts that are proportionate to the woman's body. Breast reduction surgery is often used in women with large, heavy breasts, who experience significant discomfort including neck pain, back pain and numbness 
or weakness due to the weight of the breasts. During this procedure, excess skin, fat and breast tissue are removed. After surgery, breast reduction can cause a change in breast sensation as well as the inability to breastfeed.

The procedure recreates a breast with the desired appearance, contour and volume. The nipple and areola also are recreated. Normal breast sensation and normal breast function, as with nursing do not usually return when the sensory nerves or milk glands and ducts have been removed or significantly injured.

\section{Preoperative}

The most important treatment plan for reduction mammoplasty is established with preoperative photographs of the oversized breasts. Next is the marking from the sternal notch to nipple distances and the nipple to inframammary fold distances.

The patient is instructed about the purposes of the breast reduction surgery, the achievable corrections, the expected final size, shape and contour of the reduced breasts, the expected final appearance of the breast

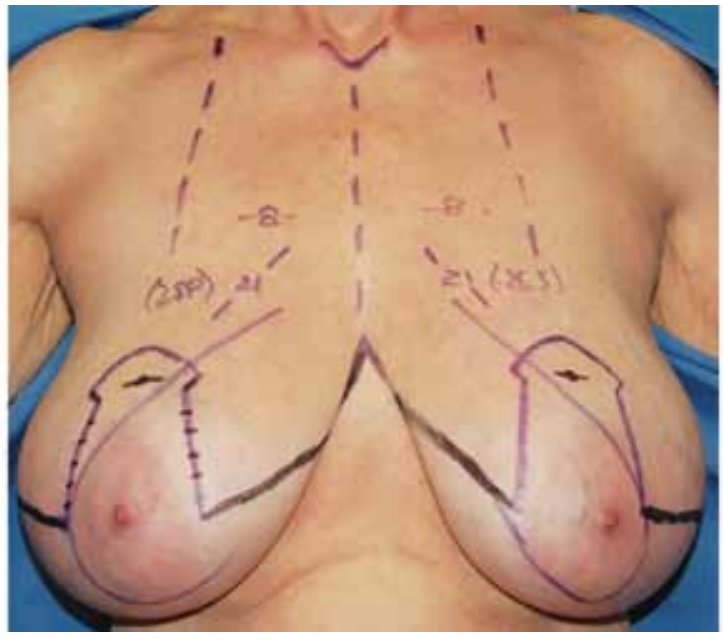

Fig. 1: Markings for breast reduction reduction scars, possible changes in the sensation of the nipple-areolar complex (NAC), possible changes in her breastfeeding capability and possible medical complications.

\section{Reduction Mammoplasty- Inferior Pedicle Technique}

The patient is laid supine upon the operating table so that the surgeon can later raise her to a sitting position that will allow visual comparison of the drape of the breasts, and an accurate assessment of the postoperative symmetry of the reduced and lifted bust. Typically, incisions are around the nipple, from the nipple down to the chest wall, and side to side under the breasts (Figs 1 and 2A). The incisions are designed so the scars will not be visible while wearing normal clothing (Fig. 2B).

Afterward, the pedicle epidermis surrounding the NAC is cut, and adipose tissue is removed from the breast. The medial, lower and lateral segments of the breast are removed, by undermining the skin below the lower curved line. Then, the NAC is transposed higher upon the breast hemisphere. The pillars of parenchymal tissue are approximated, and the skin envelope is sutured.

In this procedure, the blood supply for the NAC is maintained from an inferior centrally-based attachment to the chest wall. The skin pedicle also maintains the innervation to the NAC producing a reduced but sensitive and fully functional breast. The incision being periareolar extends downward and follows the natural curve of the breast hemisphere. After excision of the required quantity of breast tissue, NAC is transposed higher up on the breast hemisphere thereby it produces an elevated bust. The skin is then closed around the new breast mound. A small tube is kept in each breast to help drain blood and fluid for the first few days. Gauze is placed over the incisions, and

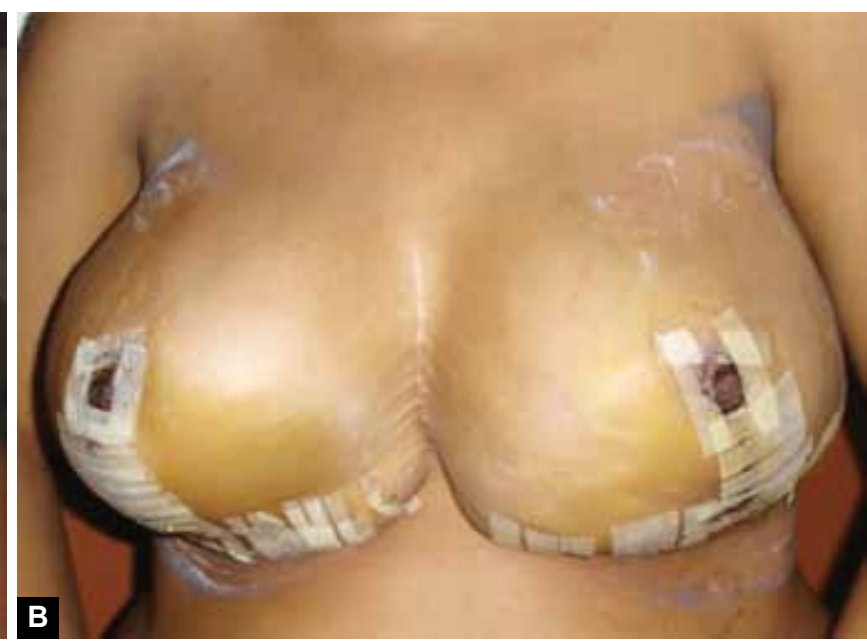

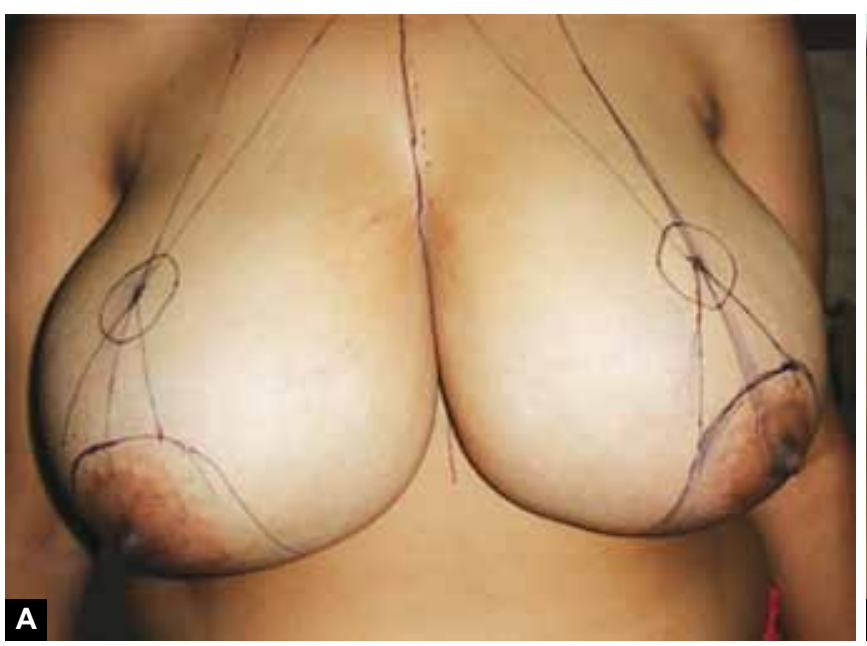

Figs 2A and B: Virginal hypertrophy: (A) Preoperative and (B) postoperative views 

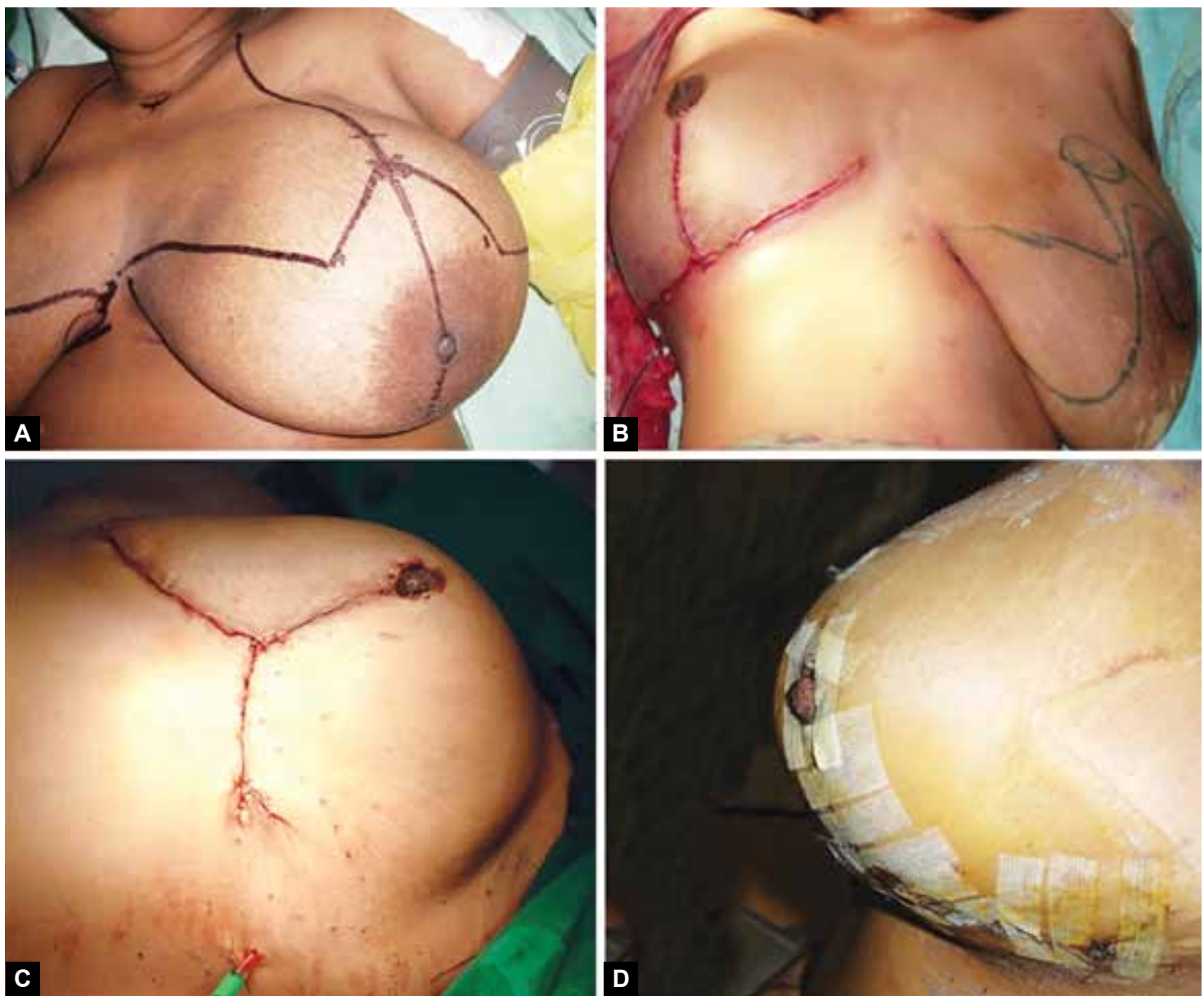

Figs 3A to D: (A) Virginal hypertrophy, (B) peroperative showing the amount of breast tissue removed from right breast, (C) final scars and (D) final side view

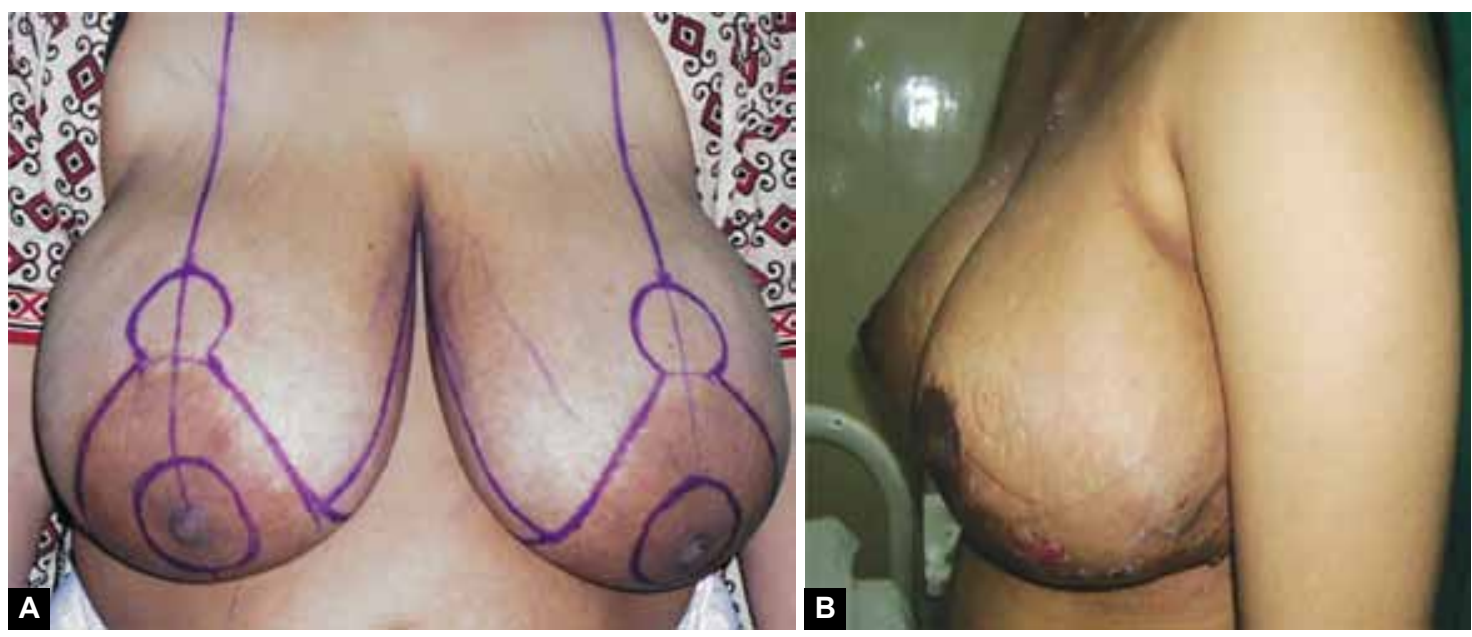

Figs 4A and B: (A) Postpartum hypertrophy and (B) postoperative side view

the breasts are wrapped in an elastic bandage and supported with a special surgical bra. Stitches are removed in 2 weeks time. Most patients have breast pain for the first few days after surgery and then mild discomfort for 2 to 3 weeks. Medicine can help to relieve the pain. Swelling and bruising may last for several weeks. Wearing a surgical bra 24 hours a day can help to reduce swelling and support the breasts while they heal.

\section{Breast Lift (Mastopexy)}

In some women, the skin is not strong or resilient enough to support the weight of the breast, causing the breasts to sag. With this condition, called ptosis, there is too much skin compared to breast tissue. To give the breast a lift, the excess skin is removed. There are several surgical techniques used, the most common breast lift operation called mastopexy repositions the nipple higher on the 

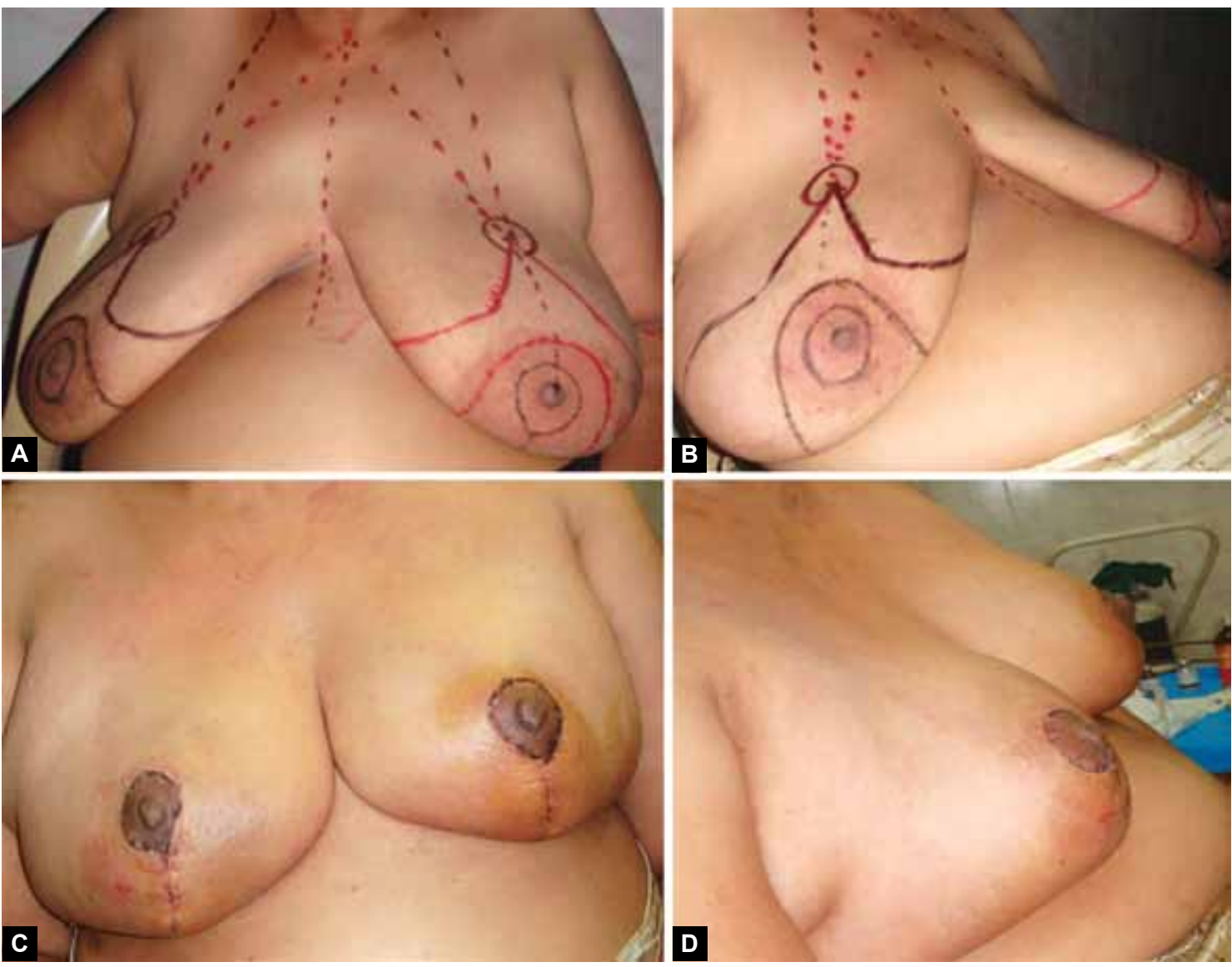

Figs 5A to D: (A) Large sagging breast postmenopausal, (B) side view of sagging breast,

(C) postoperative and (D) postoperative side view
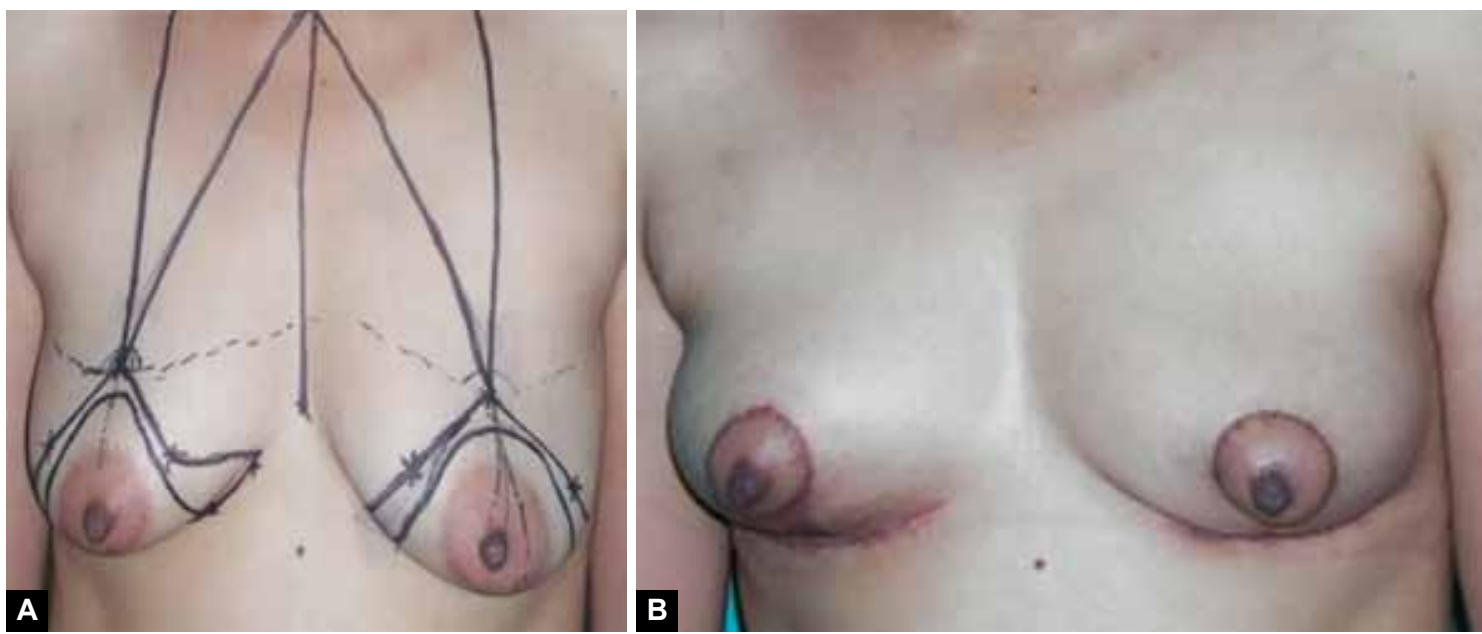

Figs 6 A and B: (A) Small sagging breast postmenopausal and (B) postoperative mastopexy

chest wall. It is similar to a breast reduction, except that only skin is removed. A breast lift can raise sagging or drooping breasts, which is a common problem with large, heavy breasts, and can elevate the nipple and areola.

\section{COMPLICATIONS}

The potential complications of the surgery can be postoperative bleeding, infection, obvious scars, irregularities in the final size and shape of the breasts or in the positioning of the areola and nipple. Others are decreased in sensation of the nipple and sometimes permanent inability to breastfeed after having the surgery. ${ }^{8}$

\section{OUR EXPERIENCE}

We present a series of five cases, two presenting with virginal hypertrophy (Figs 2 and 3), one with gestational hypertrophy (Fig. 4) and other two with postmenopausal sagging and hypertrophy (Figs 5 and 6). All the patients were evaluated to rule out other causes of breast enlargement. Four patients underwent reduction mammoplasty 
and one patient underwent mastopexy (Figs 6A and B). None of our patients complained of any of the above problems and were satisfied with the results.

\section{REFERENCES}

1. Ohlsén L, Ericsson O, Beausang-Linder M. Rapid, massive and unphysiological breast enlargement. E J Plastic Surg 1996; 19(6):307-313.

2. Swelstad, Matthew R, Swelstad, Brad B, Venkat K. Management of gestational gigantomastia. J Plastic Reconstruct Surg 2006;118(4):840-848

3. Borsetti G, Merlino G, Bergamin F. A 38 kg skin-reducing bilateral mastectomy: a unique case. JPRAS 2008;62:133-144.
4. Fiumara, Linda, Gault, David T. Massive bilateral breast reduction in an 11-year-old girl: $24 \%$ ablation of body weight. J Plastic, Reconstruct Aesthetic Surg 2009;62(8): 263-266.

5. Corriveau S, Jacobs JS. Macromastia in adolescence. Clin Plast Surg 1990;17:151-160.

6. Mayl N, Vasconez LO, Jurkiewicz MJ. Treatment of macromastia in the actively enlarging breast. Plast Reconstr Surg 1974;54:6-12.

7. Fisher W, Smith JW. Macromastia during puberty. Plast Reconstr Surg 1971;47:445-451.

8. Ship AG, Shulman J. Virginal and gravid mammary gigantismship recurrence after reduction mammoplasty. Br J Plast Surg 1971;24:396-401. 